\title{
A METHOD OF COMPOSITION ORTHOGONALITY AND NEW SEQUENCES OF ORTHOGONAL POLYNOMIALS AND FUNCTIONS FOR NON-CLASSICAL WEIGHTS
}

\author{
S. YAKUBOVICH \\ DEPARTMENT OF MATHEMATICS, FAC. SCIENCES OF UNIVERSITY OF PORTO, \\ RUA DO CAMPO ALEGRE, 687; 4169-007 PORTO (PORTUGAL)
}

\begin{abstract}
A new method of composition orthogonality is introduced. It is applied to generate new sequences of orthogonal polynomials and functions. In particular, classical orthogonal polynomials are interpreted in the sense of composition orthogonality. Finally, new sequences of orthogonal polynomials with respect to the weight function $\rho_{v}^{2}(x), \rho_{v}(x)=2 x^{v / 2} K_{v}(2 \sqrt{x}), x>0, v \geq 0$, where $K_{v}(z)$ is the modified Bessel function or Macdonald function, are investigated. Differential properties, recurrence relations, explicit representations, generating functions and Rodrigues-type formulae are obtained. The corresponding multiple orthogonal polynomials are exhibited.
\end{abstract}

\section{INTRODUCTION AND PRELIMINARY RESULTS}

In studying Prudnikov's sequence of orthogonal polynomials (see [10]) with the weight function $x^{\alpha} \rho_{v}(x)$, where $\rho_{v}(x)=2 x^{v / 2} K_{v}(2 \sqrt{x}), x>0, v \geq 0, \alpha>-1$ and $K_{v}(z)$ is the Macdonald or modified Bessel function [1], Vol. II the author interpreted this sequence in terms of the Laguerre composition orthogonality, involving the differential operator $\theta=x D x$, where $D=d / d x$. The main aim of this paper is to extend the method, employing classical orthogonal polynomials (Hermite, Laguerre, Jacobi) [1] to find new sequences of orthogonal polynomials with non-classical weights such as, for instance, the square of Macdonald function and other hypergeometric functions. In fact, we will define the composition orthogonality in the following way.

Definition 1. Let $\omega(t), \varphi(t) t \in[a, b],-\infty \leq a<b \leq \infty$ be nonnegative functions, $\theta=t$ Dt. Let $f, g$ be complex-valued functions such that the product of two operators $f(\theta) g(\theta)$ commutes. Then $f, g$ are compositionally orthogonal with respect to the measure $\omega(t) d t$ relatively to the function $\varphi$ if

$$
\int_{a}^{b} f(\theta) g(\theta)\{\varphi(t)\} \omega(t) d t=0 .
$$

We will see in the sequel that this definition suits well with the vector space of polynomials over $\mathbb{R}$, when the composition orthonormality of the sequence $\left\{P_{n}\right\}_{n \geq 0}$ is defined by

$$
\int_{a}^{b} P_{n}(\theta) P_{m}(\theta)\{\varphi(t)\} \omega(t) d t=\delta_{n, m},
$$

Date: March 5, 2021.

2000 Mathematics Subject Classification. 33C10, 42C05, 44A15.

Key words and phrases. Classical orthogonal polynomials, Macdonald function, Tricomi function, generalized hypergeometric function.

E-mail: syakubov@fc.up.pt.

The work was partially supported by CMUP, which is financed by national funds through FCT(Portugal), under the project with reference UIDB/00144/2020. 
where $\delta_{n, m}, n, m \in \mathbb{N}_{0}$ is the Kronecker symbol. Moreover, for some class of functions $\varphi$ it is possible to transform the left-hand side of the equality (1.2) to the usual orthogonality with respect to a new weight function. Then since the $n$-th power of the operator $\theta$ satisfies the Viskov-type identity [8]

$$
\theta^{n}=(x D x)^{n}=x^{n} D^{n} x^{n}, \quad n \in \mathbb{N}_{0},
$$

we can get via integration by parts new properties of the sequence $\left\{P_{n}\right\}_{n \geq 0}$ and its relationship, for instance, with classical orthogonal polynomials. In fact, let us consider a class of functions $\varphi$ representable by the modified Laplace transform of some nonnegative function $\psi$

$$
\varphi(t)=\frac{1}{t} \int_{0}^{\infty} e^{-x / t} \psi(x) d x, t \in[a, b] \subset(0, \infty) .
$$

Hence it is easily seen that

$$
\theta^{k}\left\{t^{-1} e^{-x / t}\right\}=(t D t)^{k}\left\{t^{-1} e^{-x / t}\right\}=x^{k} t^{-1} e^{-x / t}, \quad k \in \mathbb{N}_{0},
$$

and therefore, differentiating under the integral sign in (1.4), we derive

$$
\theta^{k}\{\varphi(t)\}=\frac{1}{t} \int_{0}^{\infty} e^{-x / t} x^{k} \psi(x) d x, \quad k \in \mathbb{N}_{0} .
$$

It is indeed allowed, for instance, due to the assumed convergence of the integral

$$
\int_{0}^{\infty} e^{-x / b} x^{k} \psi(x) d x<\infty, \quad k \in \mathbb{N}_{0} .
$$

Consequently, returning to (1.2) we write its left-hand side in the form

$$
\int_{a}^{b} P_{n}(\theta) P_{m}(\theta)\{\varphi(t)\} \omega(t) d t=\int_{a}^{b} \int_{0}^{\infty} e^{-x / t} P_{n}(x) P_{m}(x) \psi(x) \omega(t) \frac{d x d t}{t} .
$$

The interchange of the order of integration on the right-hand side in (1.8) is permitted by Fubini's theorem under the imposed condition

$$
\int_{a}^{b} \int_{0}^{\infty} e^{-x / t} x^{k} \psi(x) \omega(t) \frac{d x d t}{t}<\infty, \quad k \in \mathbb{N}_{0},
$$

and, combining with (1.2), we find the equalities

$$
\int_{a}^{b} P_{n}(\theta) P_{m}(\theta)\{\varphi(t)\} \omega(t) d t=\int_{0}^{\infty} P_{n}(x) P_{m}(x) \psi(x) \Omega(x) d x=\delta_{n, m},
$$

where

$$
\Omega(x)=\int_{a}^{b} e^{-x / t} \omega(t) \frac{d t}{t}, \quad x>0 .
$$

Thus we see that the sequence $\left\{P_{n}\right\}_{n \geq 0}$ is orthonormal over $(0, \infty)$ with respect to the measure $\Omega(x) d x$. Moreover, one can consider the left-hand side of the first equality in (1.10) as an inner product on the vector space of polynomials over $\mathbb{R}$ (a pre-Hilbert space)

$$
\langle p, q\rangle=\int_{a}^{b} p(\theta) q(\theta)\{\varphi(t)\} \omega(t) d t,
$$

inducing the norm by the equality 


$$
\|p\|=\sqrt{\langle p, p\rangle}=\left(\int_{0}^{\infty} p^{2}(x) \psi(x) \Omega(x) d x\right)^{1 / 2} .
$$

\section{THE USE OF CLASSICAL ORTHOGONAL POLYNOMIALS}

2.1. Laguerre polynomials. We begin to consider sequences of orthogonal polynomials which are compositionally orthogonal with respect to the measure $t^{v} e^{-t} d t$ over $\mathbb{R}_{+}$related to Laguerre polynomials $\left\{L_{n}^{v}\right\}_{n \geq 0}, v>$ -1 . In fact, letting $\omega(t)=t^{v} e^{-t}, t>0$ in (1.2), we get

$$
\int_{0}^{\infty} P_{n}(\theta) P_{m}(\theta)\{\varphi(t)\} t^{v} e^{-t} d t=\delta_{n, m} .
$$

The corresponding integral (1.11) is calculated in [1], Vol. II, and we obtain

$$
\Omega(x)=\int_{0}^{\infty} e^{-x / t-t} t^{v-1} d t=2 x^{v / 2} K_{v}(2 \sqrt{x}) \equiv \rho_{v}(x), x>0,
$$

where $K_{v}(z)$ is the modified Bessel function or Macdonald function [9]. The function $\rho_{v}$ has the MellinBarnes integral representation in the form (cf. [10])

$$
\rho_{v}(x)=\frac{1}{2 \pi i} \int_{\gamma-i \infty}^{\gamma+i \infty} \Gamma(v+s) \Gamma(s) x^{-s} d s, \quad x, \gamma \in \mathbb{R}_{+}, v \in \mathbb{R},
$$

where $\Gamma(z)$ is Euler's gamma-function [1], Vol. I. The asymptotic behavior of the modified Bessel function at infinity and near the origin [1], Vol. II gives the corresponding values for the function $\rho_{v}, v \in \mathbb{R}$. Precisely, we have

$$
\begin{gathered}
\rho_{v}(x)=O\left(x^{(v-|v|) / 2}\right), x \rightarrow 0, v \neq 0, \quad \rho_{0}(x)=O(\log x), x \rightarrow 0, \\
\rho_{v}(x)=O\left(x^{v / 2-1 / 4} e^{-2 \sqrt{x}}\right), x \rightarrow+\infty .
\end{gathered}
$$

Therefore, if the condition (cf. (1.9))

$$
\int_{0}^{\infty} x^{k} \rho_{v}(x) \psi(x) d x<\infty, \quad k \in \mathbb{N}_{0}
$$

holds valid, we arrive at the following proposition.

Proposition 1. Let $v>-1, \varphi, \psi$ be nonnegative functions defined on $\mathbb{R}_{+}$which are related by the modified Laplace transform (1.4). Then under condition (2.6) the finiteness of the integral

$$
\int_{0}^{\infty} x^{k} e^{-x / M} \psi(x) d x<\infty, \quad k \in \mathbb{N}_{0}
$$

for some $M>0$ the sequence $\left\{P_{n}\right\}_{n \geq 0}$ of orthogonal polynomials with respect to the measure $\rho_{v}(x) \psi(x) d x$ over $\mathbb{R}_{+}$is compositionally orthogonal in the sense of Laguerre relatively to the function $\varphi$, i.e.

$$
\int_{0}^{\infty} P_{n}(\theta) P_{m}(\theta)\{\varphi(t)\} t^{v} e^{-t} d t=\int_{0}^{\infty} P_{n}(x) P_{m}(x) \rho_{v}(x) \psi(x) d x=\delta_{n, m} .
$$

Proof. Since via (2.7) the integral

$$
\frac{1}{t} \int_{0}^{\infty} e^{-x / t} x^{k} \psi(x) d x, \quad k \in \mathbb{N}_{0}
$$

converges uniformly with respect to $t \in[1 / M, M], M>0$, the consecutive differentiation under the integral sign is allowed, and we derive, recalling (1.6) 
Then

$$
\begin{gathered}
P_{n}(\theta) P_{m}(\theta)\{\varphi(t)\}=P_{n}(\theta) P_{m}(\theta)\left\{\frac{1}{t} \int_{0}^{\infty} e^{-x / t} \psi(x) d x\right\} \\
=\frac{1}{t} \int_{0}^{\infty} e^{-x / t} P_{n}(x) P_{m}(x) \psi(x) d x .
\end{gathered}
$$

$$
\begin{gathered}
\int_{0}^{\infty} P_{n}(\theta) P_{m}(\theta)\{\varphi(t)\} t^{v} e^{-t} d t=\lim _{M \rightarrow \infty} \int_{1 / M}^{M} \int_{0}^{\infty} e^{-x / t} P_{n}(x) P_{m}(x) \psi(x) t^{v-1} e^{-t} d x d t \\
=\int_{0}^{\infty} P_{n}(x) P_{m}(x) \rho_{v}(x) \psi(x) d x=\delta_{n, m},
\end{gathered}
$$

where the latter equality is guaranteed by condition (2.6) and Fubini's theorem.

Remark 1. Letting $v \geq 0, \psi(x)=x^{\alpha}, \alpha>-1$, we find the sequence $\left\{P_{n}^{v, \alpha}\right\}_{n \geq 0}$ of Prudnikov's orthogonal polynomials studied in [10]

$$
\int_{0}^{\infty} P_{n}^{v, \alpha}(x) P_{m}^{v, \alpha}(x) \rho_{v}(x) x^{\alpha} d x=\Gamma(\alpha+1) \int_{0}^{\infty} P_{n}(\theta) P_{m}(\theta)\left\{t^{\alpha}\right\} t^{v} e^{-t} d t=\delta_{n, m} .
$$

2.2. Hermite polynomials. Other interesting case is the Hermite orthogonality with respect to the measure $e^{-t^{2}} d t$ over $\mathbb{R}$. Our method will work on the following even extension of the modified Laplace transform (1.4)

$$
\varphi(|t|)=\frac{1}{|t|} \int_{0}^{\infty} e^{-x /|t|} \psi(x) d x, t \in \mathbb{R} \backslash\{0\} .
$$

Hence when the Parseval identity for the Mellin transform [6] suggests the equality

$$
\int_{0}^{\infty} e^{-x / t-t^{2}} \frac{d t}{t}=\frac{1}{4 \pi i} \int_{\gamma-i \infty}^{\gamma+i \infty} \Gamma\left(\frac{s}{2}\right) \Gamma(s) x^{-s} d s, \quad x, \gamma>0 .
$$

Invoking the duplication formula for the gamma function [1], we get the right-hand side of (2.11) in the form

$$
\begin{aligned}
\frac{1}{4 \pi i} \int_{\gamma-i \infty}^{\gamma+i \infty} \Gamma\left(\frac{s}{2}\right) \Gamma(s) x^{-s} d s & =\frac{1}{4 \pi^{3 / 2} i} \int_{\gamma / 2-i \infty}^{\gamma / 2+i \infty} \Gamma^{2}(s) \Gamma\left(\frac{1}{2}+s\right)\left(\frac{x}{2}\right)^{-2 s} d s \\
= & \frac{1}{2 \sqrt{\pi}} \rho_{1 / 2,2}\left(\frac{x^{2}}{4}\right),
\end{aligned}
$$

where by $\rho_{v, k}(x)$ we denote the ultra-exponential weight function introduced in [10]

$$
\rho_{v, k}(x)=\frac{1}{2 \pi i} \int_{\gamma-i \infty}^{\gamma+i \infty} \Gamma^{k}(s) \Gamma(v+s) x^{-s} d s, x, v, \gamma>0, k \in \mathbb{N}_{0} .
$$

Proposition 2. Let $t>0, x \in \mathbb{R}, \varphi(t), \psi(x)$ be nonnegative functions which are related by (2.10). If $\psi$ is even then under conditions (2.7) and

$$
\int_{-\infty}^{\infty}|x|^{k} \rho_{1 / 2,2}\left(\frac{x^{2}}{4}\right) \psi(x) d x<\infty, \quad k \in \mathbb{N}_{0}
$$

the sequence $\left\{P_{n}\right\}_{n \geq 0}$ of orthogonal polynomials with respect to the measure $2^{-1} \pi^{-1 / 2} \rho_{1 / 2,2}\left(x^{2} / 4\right) \psi(x) d x$ over $\mathbb{R}$ is compositionally orthogonal in the sense of Hermite relatively to the function $\varphi(|t|)$, i.e. 


$$
\int_{-\infty}^{\infty} P_{n}(\theta) P_{m}(\theta)\{\varphi(|t|)\} e^{-t^{2}} d t=\int_{-\infty}^{\infty} P_{n}(x) P_{m}(x) \rho_{1 / 2,2}\left(\frac{x^{2}}{4}\right) \psi(x) \frac{d x}{\sqrt{\pi}}=\delta_{n, m} .
$$

Proof. Indeed, since

$$
\int_{-\infty}^{\infty} P_{n}(\theta) P_{m}(\theta)\{\varphi(|t|)\} e^{-t^{2}} d t=\int_{0}^{\infty}\left[P_{n}(\theta) P_{m}(\theta)+P_{n}(-\theta) P_{m}(-\theta)\right]\{\varphi(t)\} e^{-t^{2}} d t
$$

we have due to (1.4), (1.6), (2.7)

$$
\left[P_{n}(\theta) P_{m}(\theta)+P_{n}(-\theta) P_{m}(-\theta)\right]\{\varphi(t)\}=\frac{1}{t} \int_{0}^{\infty} e^{-x / t}\left[P_{n}(x) P_{m}(x)+P_{n}(-x) P_{m}(-x)\right] \psi(x) d x .
$$

Thus appealing to (2.11), (2.12), (2.14) and Fubini's theorem, we obtain finally from (2.16)

$$
\begin{gathered}
\int_{0}^{\infty} \int_{0}^{\infty} e^{-x / t-t^{2}}\left[P_{n}(x) P_{m}(x)+P_{n}(-x) P_{m}(-x)\right] \psi(x) \frac{d x d t}{t} \\
=\int_{0}^{\infty}\left[P_{n}(x) P_{m}(x)+P_{n}(-x) P_{m}(-x)\right] \rho_{1 / 2,2}\left(\frac{x^{2}}{4}\right) \psi(x) \frac{d x}{\sqrt{\pi}} \\
=\int_{-\infty}^{\infty} P_{n}(x) P_{m}(x) \rho_{1 / 2,2}\left(\frac{x^{2}}{4}\right) \psi(x) \frac{d x}{2 \sqrt{\pi}}=\delta_{n, m} .
\end{gathered}
$$

2.3. Jacobi polynomials. The modified sequence $\left\{P_{n}^{\alpha, \beta}(2 t-1)\right\}_{n \geq 0}$ of these classical polynomials is orthogonal with respect to the measure $(1-t)^{\alpha} t^{\beta} d t, \alpha, \beta>-1$ over the interval $[0,1]$. Therefore the kernel $\Omega(x), x>0(1.11)$ is calculated accordingly, and we obtain

$$
\Omega(x)=\int_{0}^{1}(1-t)^{\alpha} t^{\beta-1} e^{-x / t} d t=e^{-x} \int_{0}^{\infty} t^{\alpha}(1+t)^{-\alpha-\beta-1} e^{-x t} d t=\Gamma(1+\alpha) e^{-x} U(1+\alpha, 1-\beta, x),
$$

where $U(a, b, z)$ is the Tricomi function [4]. Hence we arrive at

Proposition 3. Let $\alpha>-1, \beta>0, \varphi, \psi$ be nonnegative functions defined on $\mathbb{R}_{+}$which are related by the modified Laplace transform (1.4). Then under the condition

$$
\int_{0}^{\infty} x^{k} e^{-x} \psi(x) d x<\infty, \quad k \in \mathbb{N}_{0}
$$

the sequence $\left\{P_{n}\right\}_{n \geq 0}$ of orthogonal polynomials with respect to the measure $e^{-x} U(1+\alpha, 1-\beta, x) \psi(x) d x$ over $\mathbb{R}_{+}$is compositionally orthogonal in the sense of Jacobi relatively to the function $\varphi$, i.e.

$$
\int_{0}^{1} P_{n}(\theta) P_{m}(\theta)\{\varphi(t)\}(1-t)^{\alpha} t^{\beta} d t=\Gamma(1+\alpha) \int_{0}^{\infty} P_{n}(x) P_{m}(x) e^{-x} U(1+\alpha, 1-\beta, x) \psi(x) d x=\delta_{n, m} .
$$

Proof. Indeed, since

$$
P_{n}(\theta) P_{m}(\theta)\{\varphi(t)\}=P_{n}(\theta) P_{m}(\theta)\left\{\frac{1}{t} \int_{0}^{\infty} e^{-x / t} \psi(x) d x\right\}=\frac{1}{t} \int_{0}^{\infty} e^{-x / t} P_{n}(x) P_{m}(x) \psi(x) d x,
$$

where the consecutive differentiation under the integral sign is permitted owing to the estimate 


$$
\frac{1}{t} \int_{0}^{\infty} e^{-x / t} x^{k} \psi(x) d x \leq \frac{1}{\delta} \int_{0}^{\infty} e^{-x} x^{k} \psi(x) d x, 0<\delta \leq t \leq 1, k \in \mathbb{N}_{0}
$$

and the latter integral by $x$ is finite via (2.18). Hence, taking into account (2.17),

$$
\begin{gathered}
\int_{0}^{1} P_{n}(\theta) P_{m}(\theta)\{\varphi(t)\}(1-t)^{\alpha} t^{\beta} d t=\lim _{\delta \rightarrow 0+} \int_{\delta}^{1} P_{n}(\theta) P_{m}(\theta)\{\varphi(t)\}(1-t)^{\alpha} t^{\beta} d t \\
=\lim _{\delta \rightarrow 0+} \int_{\delta}^{1} \int_{0}^{\infty} e^{-x / t} P_{n}(x) P_{m}(x) \psi(x)(1-t)^{\alpha} t^{\beta-1} d x d t \\
=\int_{0}^{1} \int_{0}^{\infty} e^{-x / t} P_{n}(x) P_{m}(x) \psi(x)(1-t)^{\alpha} t^{\beta-1} d x d t \\
=\Gamma(1+\alpha) \int_{0}^{\infty} P_{n}(x) P_{m}(x) e^{-x} U(1+\alpha, 1-\beta, x) \psi(x) d x=\delta_{n, m},
\end{gathered}
$$

where the interchange of the order of integration is possible by Fubini's theorem due to (2.18) and an elementary estimate of the Tricomi function

$$
\Gamma(1+\alpha) U(1+\alpha, 1-\beta, x)=\int_{0}^{\infty} t^{\alpha}(1+t)^{-\alpha-\beta-1} e^{-x t} d t \leq \int_{0}^{\infty} t^{\alpha}(1+t)^{-\alpha-\beta-1} d t=B(1+\alpha, \beta),
$$

where $B(a, b)$ is the Euler beta function [1], Vol. I.

\section{Properties of Prudnikov's Weight FUnCtions AND their PROdUCts}

In this section we will exhibit properties of the weight functions $\rho_{v+1}(x) \rho_{v}(x), \rho_{v}^{2}(x), x>0$, where $\rho_{v}(x)$ is defined by (2.2), (2.3), in order to involve them in the sequel to investigate the corresponding orthogonal and multiple orthogonal polynomials. In particular, we will establish their differential properties, integral representations and differential equations. Concerning the function $\rho_{v}$, we found in [10] the following integral representation in terms of Laguerre polynomials

$$
\frac{(-1)^{n} x^{n}}{n !} \rho_{v}(x)=\int_{0}^{\infty} t^{v+n-1} e^{-t-x / t} L_{n}^{v}(t) d t, \quad n \in \mathbb{N}_{0}
$$

It has a relationship with the Riemann-Liouville fractional integral [9]

$$
\left(I_{-}^{\alpha} f\right)(x) \equiv\left(I_{-}^{\alpha} f(x)\right)=\frac{1}{\Gamma(\alpha)} \int_{x}^{\infty}(t-x)^{\alpha-1} f(t) d t, \quad \operatorname{Re} \alpha>0
$$

namely, we get the formula

$$
\rho_{v}(x)=\left(I_{-}^{v} \rho_{0}\right)(x), v>0 .
$$

Further, the index law for fractional integrals immediately implies

$$
\rho_{v+\mu}(x)=\left(I_{-}^{v} \rho_{\mu}\right)(x)=\left(I_{-}^{\mu} \rho_{v}\right)(x) .
$$

The corresponding definition of the fractional derivative presumes the relation $D_{-}^{\mu}=-D I_{-}^{1-\mu}$. Hence for the ordinary $n$-th derivative of $\rho_{v}$ we find

$$
D^{n} \rho_{v}(x)=(-1)^{n} \rho_{v-n}(x), \quad n \in \mathbb{N}_{0} .
$$

The function $\rho_{v}$ possesses the following recurrence relation (see [10]) 


$$
\rho_{v+1}(x)=v \rho_{v}(x)+x \rho_{v-1}(x), \quad v \in \mathbb{R} .
$$

In the operator form it can be written as follows

$$
\rho_{v+1}(x)=(v-x D) \rho_{v}(x) .
$$

Now we will derive the Mellin-Barnes representations for the product of functions $\rho_{v+1} \rho_{v}$ and for the square $\rho_{v}^{2}$. In fact, it can be done, using the related formulas for the product of Macdonald functions. Thus, appealing to Entries 8.4.23.31, 8.4.23.27 in [5], Vol. III, we obtain

$$
\begin{gathered}
\rho_{v+1}(x) \rho_{v}(x)=\frac{4^{-v}}{2 i \sqrt{\pi}} \int_{\gamma-i \infty}^{\gamma+i \infty} \frac{\Gamma(s+2 v+1) \Gamma(s+v) \Gamma(s)}{\Gamma(s+v+1 / 2)}(4 x)^{-s} d s, \quad x, \gamma>0, \\
\rho_{v}^{2}(x)=\frac{4^{-v}}{i \sqrt{\pi}} \int_{\gamma-i \infty}^{\gamma+i \infty} \frac{\Gamma(s+2 v) \Gamma(s+v) \Gamma(s)}{\Gamma(s+v+1 / 2)}(4 x)^{-s} d s, \quad x, \gamma>0 .
\end{gathered}
$$

Immediate consequences of these formulas are relationships of the products of Prudnikov's weight functions $\rho_{v+1} \rho_{v}, \rho_{v}^{2}$ with $\rho_{2 v+1}, \rho_{2 v}$, correspondingly. Indeed, it can be done via Entry 8.4.2.3 in [5], Vol. III and the Parseval equality for the Mellin transform. Hence we deduce from (3.8), (3.9), respectively,

$$
\begin{gathered}
\rho_{v+1}(x) \rho_{v}(x)=4^{-v} \int_{0}^{1}(1-t)^{-1 / 2} t^{v-1} \rho_{2 v+1}\left(\frac{4 x}{t}\right) d t \\
=\frac{x^{v} \sqrt{\pi}}{\Gamma(v+1 / 2)} \int_{0}^{1}(1-t)^{v-1 / 2} t^{-v-1} \rho_{v+1}\left(\frac{4 x}{t}\right) d t \\
\rho_{v}^{2}(x)=2^{1-2 v} \int_{0}^{1}(1-t)^{-1 / 2} t^{v-1} \rho_{2 v}\left(\frac{4 x}{t}\right) d t \\
=\frac{2 x^{v} \sqrt{\pi}}{\Gamma(v+1 / 2)} \int_{0}^{1}(1-t)^{v-1 / 2} t^{-v-1} \rho_{v}\left(\frac{4 x}{t}\right) d t .
\end{gathered}
$$

An ordinary differential equation for the function $\rho_{v+1} \rho_{v}$ is given by

Proposition 4. The function $u_{v}=\rho_{v+1} \rho_{v}$ satisfies the following third order differential equation

$$
x^{2} \frac{d^{3} u_{v}}{d x^{3}}+x(2-3 v) \frac{d^{2} u_{v}}{d x^{2}}+2(v(v-1)-2 x) \frac{d u_{v}}{d x}+2(2 v-1) u_{v}(x)=0 .
$$

Proof. Differentiating $u_{v}$ with the use of (3.5), we have

$$
\frac{d u_{v}}{d x}=-\rho_{v}^{2}(x)-\rho_{v+1}(x) \rho_{v-1}(x) .
$$

Multiplying both sides of (3.13) by $x$ and employing the recurrence relation (3.6), we get

$$
x \frac{d u_{v}}{d x}=-x \rho_{v}^{2}(x)+v \rho_{v+1}(x) \rho_{v}(x)-\rho_{v+1}^{2}(x) .
$$

Differentiating both sides of the latter equality, using (3.6), (3.13) and the notation $u_{v}=\rho_{v+1} \rho_{v}$, we obtain

$$
\frac{d}{d x}\left(x \frac{d u_{v}}{d x}\right)=-(1+2 v) \rho_{v}^{2}(x)+v \frac{d u_{v}}{d x}+4 u_{v}(x) .
$$

Differentiating (3.14) and multiplying the result by $x$, we recall (3.6) to find 


$$
x \frac{d^{2}}{d x^{2}}\left(x \frac{d u_{v}}{d x}\right)=-2 v(1+2 v) \rho_{v}^{2}(x)+v x \frac{d^{2} u_{v}}{d x^{2}}+4 x \frac{d u_{v}}{d x}+2(1+2 v) u_{v}(x) .
$$

Hence, expressing $(1+2 v) \rho_{v}^{2}(x)$ from (3.14) and fulfilling the differentiation on the left-hand side of (3.15), we get (3.12).

Concerning the differential equation for the function $\rho_{v}^{2}$, we prove

Proposition 5. The function $h_{v}=\rho_{v}^{2}$ satisfies the following third order differential equation

$$
x^{2} \frac{d^{3} h_{v}}{d x^{3}}+3 x(1-v) \frac{d^{2} h_{v}}{d x^{2}}+\left(2 v^{2}+1-3 v-4 x\right) \frac{d h_{v}}{d x}+2(2 v-1) h_{v}(x)=0 .
$$

Proof. Since $x\left(\rho_{v}^{2}(x)\right)^{\prime}=-2 x \rho_{v-1}(x) \rho_{v}(x)=2 v \rho_{v}^{2}(x)-2 \rho_{v+1}(x) \rho_{v}(x)=2 v h_{v}(x)-2 \rho_{v+1}(x) \rho_{v}(x)$ we derive, owing to (3.5), (3.6)

$$
\begin{gathered}
x \frac{d}{d x}\left(x \frac{d h_{v}}{d x}-2 v h_{v}(x)\right)=-2 x \frac{d}{d x}\left(\rho_{v+1}(x) \rho_{v}(x)\right) \\
=2 x h_{v}(x)-2 v \rho_{v+1}(x) \rho_{v}(x)+2 \rho_{v+1}^{2}(x)=2\left(x-v^{2}\right) h_{v}(x)+v x \frac{d h_{v}}{d x}+2 \rho_{v+1}^{2}(x) .
\end{gathered}
$$

Hence one more differentiation yields

$$
\frac{d}{d x}\left(x \frac{d}{d x}\left(x \frac{d h_{v}}{d x}-2 v h_{v}(x)\right)\right)=\frac{d}{d x}\left(2\left(x-v^{2}\right) h_{v}(x)+v x \frac{d h_{v}}{d x}\right)-4 \rho_{v+1}(x) \rho_{v}(x) .
$$

But from the beginning of the proof we find

$$
2 \rho_{v+1}(x) \rho_{v}(x)=2 v h_{v}(x)-x \frac{d h_{v}}{d x} .
$$

Therefore, substituting this expression into the previous equality, we fulfil the differentiation to arrive at (3.16) and to complete the proof.

Corollary 1. The following recurrence relations between functions $u_{v}, h_{v}$ hold

$$
\begin{gathered}
u_{v}=v h_{v}+x u_{v-1}, \\
h_{v+1}=v^{2} h_{v}+2 x v u_{v-1}+x^{2} h_{v-1}=2 v u_{v}+x^{2} h_{v-1}-v^{2} h_{v} .
\end{gathered}
$$

Proof. Equality (3.18) is a direct consequence of (3.17) and (3.5). Equalities (3.19), in turn, are obtained, taking squares of both sides of (3.6) and employing (3.18). 


\section{ORThOGONAL POLYNOMIALS WiTH $\rho_{v}^{2}$ WEIGHT FUNCTION}

The object of this section is to characterize the sequence of orthogonal polynomials $\left\{P_{n}\right\}_{n \geq 0}$, satisfying the orthogonality conditions

$$
\int_{0}^{\infty} P_{m}(x) P_{n}(x) \rho_{v}^{2}(x) d x=\delta_{n, m}, \quad v>-\frac{1}{2} .
$$

Clearly, up to a normalization factor conditions (4.1) are equivalent to the equalities

$$
\int_{0}^{\infty} P_{n}(x) \rho_{v}^{2}(x) x^{m} d x=0, \quad m=0,1, \ldots, n-1, \quad n \in \mathbb{N} .
$$

The moments of the weight $\rho_{v}^{2}(x)$ can be obtained immediately from the Mellin-Barnes representation (3.9), treating it as the inverse Mellin transform [6]. Hence we get

$$
\int_{0}^{\infty} \rho_{v}^{2}(x) x^{\mu} d x=\sqrt{\pi} \frac{\Gamma(1+\mu+2 v) \Gamma(1+\mu+v) \Gamma(1+\mu)}{2^{1+2(v+\mu)} \Gamma(\mu+v+3 / 2)}, \mu>\max \{-1,-1-v, 1-2 v\} .
$$

Furthermore, the sequence $\left\{P_{n}\right\}_{n \geq 0}$ satisfies the 3 -term recurrence relation in the form

$$
x P_{n}(x)=A_{n+1} P_{n+1}(x)+B_{n} P_{n}(x)+A_{n} P_{n-1}(x),
$$

where $P_{-1}^{v}(x) \equiv 0, P_{n}(x)=\sum_{k=0}^{n} a_{n, k} x^{k}, a_{n, n} \neq 0$ and

$$
A_{n+1}=\frac{a_{n}}{a_{n+1}}, \quad B_{n}=\frac{b_{n}}{a_{n}}-\frac{b_{n+1}}{a_{n+1}}, \quad a_{n} \equiv a_{n, n}, b_{n} \equiv a_{n, n-1} .
$$

Basing on properties of the functions $\rho_{v}^{2}, \rho_{v+1} \rho_{v}$ and orthonormality (4.1), we establish

Proposition 6. Let $v>-1$. The following formulas hold

$$
\begin{gathered}
\int_{0}^{\infty} P_{n}^{2}(x) \rho_{v+1}(x) \rho_{v}(x) d x=\frac{1}{2}+v+n, \\
\int_{0}^{\infty} P_{n}^{2}(x) x \rho_{v}(x) \rho_{v-1}(x) d x=\frac{1}{2}+n \\
\int_{0}^{\infty} P_{n}^{2}(x) x^{2} \rho_{v}(x) \rho_{v-2}(x) d x=B_{n}-(v-1)\left(\frac{1}{2}+n\right), \\
\int_{0}^{\infty} P_{n}^{2}(x) \rho_{v+2}(x) \rho_{v}(x) d x=B_{n}+(v+1)\left(\frac{1}{2}+v+n\right) .
\end{gathered}
$$

Proof. In fact, recurrence relation (3.6) and orthonormality (4.1) implies

$$
\int_{0}^{\infty} P_{n}^{2}(x) \rho_{v+1}(x) \rho_{v}(x) d x=v+\int_{0}^{\infty} P_{n}^{2}(x) x \rho_{v}(x) \rho_{v-1}(x) d x
$$

Hence, integrating by parts in the latter integral and eliminating integrated terms by virtue of the asymptotic behavior (2.4), (2.5), we find

$$
\int_{0}^{\infty} P_{n}^{2}(x) x \rho_{v}(x) \rho_{v-1}(x) d x=\int_{0}^{\infty}\left(\frac{1}{2} P_{n}^{2}(x)+x P_{n}(x) P_{n}^{\prime}(x)\right) \rho_{v}^{2}(x) d x .
$$

Therefore, we have from (4.9), (4.10) and orthogonality (4.1) 


$$
\int_{0}^{\infty} P_{n}^{2}(x) \rho_{v+1}(x) \rho_{v}(x) d x=\frac{1}{2}+v+n
$$

which proves (4.5). Concerning equality (4.6), it is a direct consequence of (3.6), (4.1). The same idea is to prove (4.7), (4.8), employing (4.3) as well.

The composition orthogonality which is associated with the sequence $\left\{P_{n}\right\}_{n \geq 0}$ is given by

Theorem 1. Let $v>-1 / 2$. The sequence $\left\{P_{n}\right\}_{n>0}$ is compositionally orthogonal in the sense of Laguerre relatively to the function $t^{v} e^{t} \Gamma(-v, t)$, where $\Gamma(\mu, z)$ is the incomplete gamma function, i.e.

$$
\int_{0}^{\infty} t^{v} e^{-t} P_{n}(\theta) P_{m}(\theta)\left(t^{v} e^{t} \Gamma(-v, t)\right) d t=\frac{\delta_{n, m}}{\Gamma(1+v)} .
$$

Proof. Writing $P_{n}$ explicitly, we appeal to the integral representation (3.1) for $\rho_{v}(x)$ to write the left-hand side of (4.2) in the form

$$
\begin{aligned}
\int_{0}^{\infty} P_{n}(x) \rho_{v}^{2}(x) x^{m} d x= & (-1)^{m} m ! \sum_{k=0}^{n} a_{n, k}(-1)^{k} k ! \int_{0}^{\infty} \int_{0}^{\infty} t^{v+k-1} e^{-t-x / t} L_{k}^{v}(t) d t \\
& \times \int_{0}^{\infty} y^{v+m-1} e^{-y-x / y} L_{m}^{v}(y) d y d x .
\end{aligned}
$$

The Fubini theorem allows to interchange the order of integration in (4.12) due to the convergence of the following integral for all $r_{1}, r_{2} \in \mathbb{N}_{0}$

$$
\begin{gathered}
\int_{0}^{\infty} \int_{0}^{\infty} t^{v+k+r_{1}-1} e^{-t-x / t} d t \int_{0}^{\infty} y^{v+m+r_{2}-1} e^{-y-x / y} d y d x \\
=\int_{0}^{\infty} \int_{0}^{\infty} t^{v+k+r_{1}} y^{v+m+r_{2}} e^{-t-y} \frac{d t d y}{t+y} \leq \frac{1}{2} \int_{0}^{\infty} \int_{0}^{\infty} t^{v+k+r_{1}-1 / 2} y^{v+m+r_{2}-1 / 2} e^{-t-y} d t d y \\
=\frac{1}{2} \Gamma\left(v+k+r_{1}+1 / 2\right) \Gamma\left(v+m+r_{2}+1 / 2\right) .
\end{gathered}
$$

Therefore after integration with respect to $x$ we find from (4.12)

$$
\int_{0}^{\infty} P_{n}(x) \rho_{v}^{2}(x) x^{m} d x=(-1)^{m} m ! \sum_{k=0}^{n} a_{n, k}(-1)^{k} k ! \int_{0}^{\infty} \int_{0}^{\infty} t^{v+k} y^{v+m} e^{-t-y} L_{k}^{v}(t) L_{m}^{v}(y) \frac{d t d y}{t+y} .
$$

However the Rodrigues formula for Laguerre polynomials and Viskov-type identity (1.3) suggest to write the right-hand side of (4.13) as follows

$$
\begin{aligned}
& (-1)^{m} m ! \sum_{k=0}^{n} a_{n, k}(-1)^{k} k ! \int_{0}^{\infty} \int_{0}^{\infty} t^{v+k} y^{v+m} e^{-t-y} L_{k}^{v}(t) L_{m}^{v}(y) \frac{d t d y}{t+y} \\
& =(-1)^{m} \sum_{k=0}^{n} a_{n, k}(-1)^{k} k ! \int_{0}^{\infty} t^{v+k} e^{-t} L_{k}^{v}(t) \int_{0}^{\infty} \theta^{m}\left(y^{v} e^{-y}\right) \frac{d y d t}{t+y} .
\end{aligned}
$$

The inner integral with respect to $y$ on the right-hand side of the latter equality can be treated via integration by parts, and we obtain

$$
\int_{0}^{\infty} \theta^{m}\left(y^{v} e^{-y}\right) \frac{d y}{t+y}=(-1)^{m} \int_{0}^{\infty} y^{v} e^{-y} \theta^{m}\left(\frac{1}{t+y}\right) d y .
$$


Working out the differentiation, we find

$$
\begin{gathered}
\theta^{m}\left(\frac{1}{t+y}\right)=y^{m} \frac{d^{m}}{d y^{m}}\left(\sum_{k=0}^{m}\left(\begin{array}{c}
m \\
k
\end{array}\right)(-1)^{m-k} t^{m-k}(t+y)^{k-1}\right) \\
=(-1)^{m} t^{m} y^{m} \frac{d^{m}}{d y^{m}}\left(\frac{1}{t+y}\right)=\frac{m ! t^{m} y^{m}}{(t+y)^{m+1}} .
\end{gathered}
$$

Consequently, combining with (4.14), (4.15), equality (4.13) becomes

$$
\int_{0}^{\infty} P_{n}(x) \rho_{v}^{2}(x) x^{m} d x=m ! \sum_{k=0}^{n} a_{n, k}(-1)^{k} k ! \int_{0}^{\infty} t^{v+k+m} e^{-t} L_{k}^{v}(t) \int_{0}^{\infty} \frac{y^{v+m} e^{-y}}{(t+y)^{m+1}} d y d t .
$$

Meanwhile, the integral with respect to $y$ in (4.16) is calculated in (2.17) in terms of the Tricomi function up to a simple change of variables. Thus we get

$$
\int_{0}^{\infty} P_{n}(x) \rho_{v}^{2}(x) x^{m} d x=m ! \Gamma(v+m+1) \sum_{k=0}^{n} a_{n, k}(-1)^{k} k ! \int_{0}^{\infty} t^{2 v+k+m} e^{-t} L_{k}^{v}(t) U(v+m+1,1+v, t) d t .
$$

But appealing to the differential formula 13.3.24 in [4] for the Tricomi function, we have

$$
\begin{gathered}
m ! \Gamma(v+m+1) t^{v+m} U(v+m+1,1+v, t)=\Gamma(v+1) \theta^{m}\left(t^{v} U(1+v, 1+v, t)\right) \\
=\Gamma(v+1) \theta^{m}\left(t^{v} e^{t} \Gamma(-v, t)\right),
\end{gathered}
$$

where $\Gamma(a, z)$ is the incomplete gamma function

$$
\Gamma(a, z)=\int_{z}^{\infty} e^{-u} u^{a-1} d u .
$$

Hence, plugging this result in (4.17), recalling the Rodrigues formula for Laguerre polynomials and integrating by parts on its right-hand side, we combine with (4.2) to end up with equalities

$$
\begin{gathered}
\int_{0}^{\infty} P_{n}(x) \rho_{v}^{2}(x) x^{m} d x=\Gamma(v+1) \\
\times \int_{0}^{\infty} t^{v} e^{-t} P_{n}(\theta) \theta^{m}\left(t^{v} e^{t} \Gamma(-v, t)\right) d t=0, \quad m=0,1, \ldots, n-1, n \in \mathbb{N},
\end{gathered}
$$

which yield (4.11) and complete the proof of Theorem 1.

Further, the right-hand side of the first equality in (4.20) can be rewritten via integration by parts as follows

$$
\int_{0}^{\infty} t^{v+m} e^{-t} L_{m}^{v}(t) P_{n}(\theta)\left(t^{v} e^{t} \Gamma(-v, t)\right) d t=0, \quad m=0,1, \ldots, n-1, n \in \mathbb{N} .
$$

Then we expand the function $F_{n}(t)=\theta^{n}\left(t^{v} e^{t} \Gamma(-v, t)\right)$ in a series of Laguerre polynomials

$$
F_{n}(t)=\sum_{r=0}^{\infty} c_{n, r} L_{r}^{v}(t)
$$

where the coefficients $c_{n, r}$ are calculated in terms of the ${ }_{3} F_{2}$-hypergeometric functions at unity with the aid of Entry 3.31.12.1 in [2]. Precisely, we get via (4.18) 


$$
\begin{gathered}
c_{n, r}=\frac{r !}{\Gamma(1+v+r)} \int_{0}^{\infty} t^{v} e^{-t} L_{r}^{v}(t) \theta^{n}\left(t^{v} e^{t} \Gamma(-v, t)\right) d t \\
=\frac{r ! n !(1+v)_{n}}{\Gamma(1+v+r)} \int_{0}^{\infty} t^{2 v+n} e^{-t} L_{r}^{v}(t) U(v+n+1,1+v, t) d t \\
=\frac{(1+v)_{n}}{\Gamma(1+v)}\left[\frac{n ! r ! \Gamma(v)}{(1+v)_{r}}{ }_{3} F_{2}(n+1, r+1, v+n+1 ; 1-v, 1 ; 1)\right. \\
\left.+\Gamma(2 v+n+1) \Gamma(-v)_{3} F_{2}(v+r+1, v+n+1,2 v+n+1 ; 1+v, 1+v ; 1)\right],
\end{gathered}
$$

where $(a)_{z}$ is the Pochhammer symbol [1], Vol. I and it is valid for nonnegative integers $v$ by continuity. Hence after substitution the series (4.22) into (4.21) these orthogonality conditions take the form

$$
\int_{0}^{\infty} t^{v+m} e^{-t} L_{m}^{v}(t) \sum_{k=0}^{n} a_{n, k} \sum_{r=0}^{2 m} c_{k, r} L_{r}^{v}(t) d t=0, \quad m=0,1, \ldots, n-1, n \in \mathbb{N} .
$$

Calculating the integral in (4.24) via relation (2.19.14.8) in [5], Vol. II we obtain

$$
\begin{gathered}
d_{m, r}=\int_{0}^{\infty} t^{v+m} e^{-t} L_{m}^{v}(t) L_{r}^{v}(t) d t=\frac{(-1)^{r}}{r !}(1+v)_{r} \Gamma(1+v+m) \\
\quad{ }_{3} F_{2}(-r, v+m+1, m+1 ; 1+v, 1 ; 1) .
\end{gathered}
$$

Hence, equalities (4.24) become the linear system of $n$ algebraic equations with $n+1$ unknowns

$$
\sum_{k=0}^{n} a_{n, k} f_{k, m}=0, \quad m=0,1, \ldots, n-1, n \in \mathbb{N},
$$

where

$$
f_{k, m}=\sum_{r=0}^{2 m} c_{k, r} d_{m, r}
$$

Consequently, explicit values of the coefficients $a_{n, k}, k=1,2, \ldots, n$ can be expressed via Cramer's rule in terms of the free coefficient $a_{n, 0}$ as follows

$$
a_{n, k}=-a_{n, 0} \frac{D_{n, k}}{D_{n}}, \quad k=1, \ldots, n,
$$

where

$$
D_{n}=\left|\begin{array}{ccccc}
f_{1,0} & f_{2,0} & \ldots & \ldots & f_{n, 0} \\
f_{1,1} & \ldots & \ldots & \ldots & f_{n, 1} \\
\ldots & \ldots & \ldots & \ldots & \ldots \\
\vdots & \ddots & \ddots & \ddots & \vdots \\
f_{1, n-1} & \ldots & \ldots & \ldots & f_{n, n-1}
\end{array}\right|
$$




$$
D_{n, k}=\left|\begin{array}{ccccccc}
f_{1,0} & \ldots & f_{k-1,0} & f_{0,0} & f_{k+1,0} & \ldots & f_{n, 0} \\
f_{1,1} & \ldots & f_{k-1,1} & f_{0,1} & f_{k+1,1} & \ldots & f_{n, 1} \\
\ldots & \ldots & \ldots & \ldots & \ldots & \ldots & \ldots \\
\vdots & \ddots & \ddots & \vdots & \ddots & \ddots & \vdots \\
\vdots & \ddots & \ddots & \vdots & \ddots & \ddots & \vdots \\
\vdots & \ddots & \ddots & \vdots & \ddots & \ddots & \vdots \\
f_{1, n-1} & \ldots & f_{k-1, n-1} & f_{0, n-1} & f_{k+1,1} & \ldots & f_{n, n-1}
\end{array}\right| .
$$

The free coefficient can be determined, in turn, from the orthogonality conditions (4.1), (4.2), which imply the formula

$$
\int_{0}^{\infty} P_{n}(x) \rho_{v}^{2}(x) x^{n} d x=\frac{1}{a_{n, n}} .
$$

Therefore from (4.28) and (4.3) we derive

$$
\frac{1}{a_{n, n}}=-\frac{a_{n, 0} \sqrt{\pi}}{D_{n}} \frac{\Gamma(1+2 v) \Gamma(1+v)}{2^{1+2 v} \Gamma(v+3 / 2)} \sum_{k=0}^{n} D_{n, k}(n+k) ! \frac{(1+2 v)_{n+k}(1+v)_{n+k}}{4^{n+k}(v+3 / 2)_{n+k}},
$$

where $D_{n, 0} \equiv-D_{n}$. Hence

$$
a_{n, 0}= \pm \frac{D_{n}}{\left[D_{n, n}\right]^{1 / 2}}\left[\sqrt{\pi} \frac{\Gamma(1+2 v) \Gamma(1+v)}{2^{1+2 v} \Gamma(v+3 / 2)} \sum_{k=0}^{n} D_{n, k}(n+k) ! \frac{(1+2 v)_{n+k}(1+v)_{n+k}}{4^{n+k}(v+3 / 2)_{n+k}}\right]^{-1 / 2},
$$

where the sign can be chosen accordingly, making positive expressions under the square roots. Assuming also the positivity of the leading coefficient $a_{n, n}$ we have its value, correspondingly,

$$
a_{n, n}=\mp\left[D_{n, n}\right]^{1 / 2}\left[\sqrt{\pi} \frac{\Gamma(1+2 v) \Gamma(1+v)}{2^{1+2 v} \Gamma(v+3 / 2)} \sum_{k=0}^{n} D_{n, k}(n+k) ! \frac{(1+2 v)_{n+k}(1+v)_{n+k}}{4^{n+k}(v+3 / 2)_{n+k}}\right]^{-1 / 2} \text {. }
$$

Theorem 2. Let $v>-1 / 2$. The sequence of orthogonal polynomials $\left\{P_{n}\right\}_{n \geq 0}$ can be expressed explicitly, where the coefficients $a_{n, k}, k=1,2, \ldots, n$ are calculated by relations (4.28) and the free term $a_{n, 0}$ is defined by the equality (4.33). Besides, it satisfies the 3-term recurrence relation (4.4), where

$$
A_{n+1}=\frac{a_{n, 0} D_{n+1} D_{n, n}}{a_{n+1,0} D_{n} D_{n+1, n+1}}, \quad B_{n}=\frac{D_{n, n-1}}{D_{n, n}}-\frac{D_{n+1, n}}{D_{n+1, n+1}} .
$$

An analog of the Rodrigues formula for the sequence $\left\{P_{n}\right\}_{n \in \mathbb{N}_{0}}$ can be established, appealing to the integral representation of an arbitrary polynomial in terms of the associated polynomial of degree $2 n$ (see [10]). Hence, employing (2.2), we deduce

$$
\begin{gathered}
P_{n}(x)=\frac{1}{\rho_{v}(x)} \int_{0}^{\infty} t^{v-1} e^{-t-x / t} q_{2 n}(t) d t=\frac{1}{\rho_{v}^{2}(x)} \int_{0}^{\infty} u^{v-1} e^{-u-x / u} d u \int_{0}^{\infty} t^{v-1} e^{-t-x / t} q_{2 n}(t) d t \\
=\frac{(-1)^{n}}{\rho_{V}^{2}(x)} \frac{d^{n}}{d x^{n}} \int_{0}^{\infty} \int_{0}^{\infty}(u t)^{v+n-1} e^{-u-t-x(1 / u+1 / t)} q_{2 n}(t) \frac{d t d u}{(u+t)^{n}}
\end{gathered}
$$


where

$$
q_{2 n}(x)=\sum_{k=0}^{n} a_{n, k}(-1)^{k} k ! x^{k} L_{k}^{v}(x)
$$

and the $k$-th differentiation under the integral sign is permitted due to the estimate

$$
\begin{gathered}
\int_{0}^{\infty} \int_{0}^{\infty}(u t)^{v+k-1} e^{-u-t-x(1 / u+1 / t)}\left|q_{2 n}(t)\right| \frac{d t d u}{(u+t)^{k}} \\
\leq 2^{-k} \int_{0}^{\infty} u^{v+k / 2-1} e^{-u} d u \int_{0}^{\infty} t^{v+k / 2-1} e^{-t}\left|q_{2 n}(t)\right| d t \\
=2^{-k} \Gamma\left(v+\frac{k}{2}\right) \int_{0}^{\infty} t^{v+k / 2-1} e^{-t}\left|q_{2 n}(t)\right| d t<\infty, \quad k=1, \ldots, n .
\end{gathered}
$$

Then writing

we get from (4.36)

$$
\frac{(u t)^{n}}{(u+t)^{n}}=\frac{1}{(n-1) !} \int_{0}^{\infty} e^{-(1 / u+1 / t) y} y^{n-1} d y
$$

$$
\begin{aligned}
P_{n}(x)= & \frac{(-1)^{n}}{(n-1) ! \rho_{v}^{2}(x)} \frac{d^{n}}{d x^{n}} \int_{0}^{\infty} \int_{0}^{\infty} \int_{0}^{\infty} y^{n-1}(u t)^{v-1} e^{-u-t-(x+y) / u-(x+y) / t} q_{2 n}(t) d t d u d y \\
& =\frac{(-1)^{n}}{(n-1) ! \rho_{v}^{2}(x)} \frac{d^{n}}{d x^{n}} \int_{0}^{\infty} \int_{0}^{\infty} y^{n-1} t^{\nu-1} e^{-t-(x+y) / t} q_{2 n}(t) \rho_{v}(x+y) d t d y
\end{aligned}
$$

Then, expressing $q_{2 n}$ in terms of the Laguerre polynomials

$$
q_{2 n}(x)=\sum_{k=0}^{2 n} h_{2 n, k} L_{k}^{v}(x)
$$

where the coefficients $h_{2 n, k}$ are calculated by virtue of (4.28), (4.37) and relation (2.19.14.15) in [5], Vol. II, namely,

$$
\begin{gathered}
h_{2 n, k}=\frac{k !}{\Gamma(1+v+k)} \int_{0}^{\infty} t^{v} e^{-t} L_{k}^{v}(t) q_{2 n}(t) d t \\
=-\frac{k ! a_{n, 0}}{D_{n} \Gamma(1+v+k)} \sum_{r=0}^{n} D_{n, r}(-1)^{r} r ! \int_{0}^{\infty} t^{v+r} e^{-t} L_{k}^{v}(t) L_{r}^{v}(t) d t \\
=-\frac{a_{n, 0}}{D_{n}} \sum_{r=0}^{n} D_{n, r} r !(1+v)_{r} F_{2}(-k, 1+v+r, 1+r ; 1+v, 1 ; 1) .
\end{gathered}
$$

So,

$$
h_{2 n, k}=-\frac{a_{n, 0}}{D_{n}} \sum_{r=0}^{n} D_{n, r} r !(1+v)_{r} F_{2}(-k, 1+v+r, 1+r ; 1+v, 1 ; 1),
$$

and the values of the generalized hypergeometric function can be simplified via relations $(7.4 .4 ; 90,91,92,93)$ in [5], Vol. III. Precisely, we get for $k=0,1 \ldots, n$

$$
{ }_{3} F_{2}(-k, 1+v+r, 1+r ; 1+v, 1 ; 1)=0, \quad k>2 r,
$$




$$
\begin{gathered}
(1+v)_{r}{ }_{3} F_{2}(-k, 1+v+r, 1+r ; 1+v, 1 ; 1)=\frac{(2 r) !}{r !}, \quad k=2 r, \\
(1+v)_{r} F_{2}(-k, 1+v+r, 1+r ; 1+v, 1 ; 1)=-\frac{(2 r-1) !(v+3 r)}{(r-1) !}, \quad k=2 r-1, \\
(1+v)_{r 3} F_{2}(-k, 1+v+r, 1+r ; 1+v, 1 ; 1)=\frac{(2(r-1)) !}{2 r !}\left(2 r^{2}(2 r+v-1)(2 r-1)\right. \\
+r(r-1)(r+v-1)(r+v)), \quad k=2(r-1) .
\end{gathered}
$$

Therefore, returning to (4.38) and minding (4.39), (4.40), (4.41), (3.1), (3.2) we find

$$
\begin{aligned}
& P_{n}(x)=\frac{(-1)^{n+1} a_{n, 0}}{D_{n}(n-1) ! \rho_{v}^{2}(x)} \frac{d^{n}}{d x^{n}} \sum_{k=0}^{2 n} \sum_{r=0}^{n} D_{n, r} r !(1+v)_{r}{ }_{3} F_{2}(-k, 1+v+r, 1+r ; 1+v, 1 ; 1) \\
& \quad \times \int_{0}^{\infty} \int_{0}^{\infty} y^{n-1} t^{v-1} e^{-t-(x+y) / t} L_{k}^{v}(t) \rho_{v}(x+y) d t d y \\
& =\frac{(-1)^{n+1} a_{n, 0}}{D_{n}(n-1) ! \rho_{v}^{2}(x)} \frac{d^{n}}{d x^{n}} \sum_{k=0}^{2 n} \frac{1}{k !} \sum_{r=0}^{n} D_{n, r} r !(1+v)_{r} F_{2}(-k, 1+v+r, 1+r ; 1+v, 1 ; 1) \\
& \quad \times \int_{0}^{\infty} y^{n-1} \frac{d^{k}}{d x^{k}}\left((x+y)^{k} \rho_{v}(x+y)\right) \rho_{v}(x+y) d y \\
& =\frac{(-1)^{n+1} a_{n, 0}}{D_{n}(n-1) ! \rho_{v}^{2}(x)} \frac{d^{n}}{d x^{n}} \sum_{k=0}^{2 n} \frac{1}{k !} \sum_{r=0}^{n} D_{n, r} r !(1+v)_{r} F_{2}(-k, 1+v+r, 1+r ; 1+v, 1 ; 1) \\
& \quad \times \int_{x}^{\infty}(y-x)^{n-1} \frac{d^{k}}{d y^{k}}\left(y^{k} \rho_{v}(y)\right) \rho_{v}(y) d y=-\frac{a_{n, 0}}{D_{n} \rho_{v}(x)} \sum_{k=0}^{2 n} \frac{d^{k}}{d x^{k}}\left(x^{k} \rho_{v}(x)\right) \sum_{r=0}^{n} D_{n, r} r ! \\
& k ! \\
& =-\frac{a_{n, 0}}{D_{n} \rho_{v}(x)} \sum_{r=0}^{n} D_{n, r} r !(1+v)_{r} \sum_{k=0}^{2 r} \frac{d^{k}}{d x^{k}}\left(x^{k} \rho_{v}(x)\right) \frac{1}{k !}{ }_{3} F_{2}(-k, 1+v+r, 1+r ; 1+v, 1 ; 1)
\end{aligned}
$$

Thus it proves

Theorem 3. Let $v>-1 / 2, n \in \mathbb{N}_{0}$. Orthogonal polynomials $P_{n}$ satisfy the Rodrigues-type formula

$$
P_{n}(x)=-\frac{a_{n, 0}}{D_{n} \rho_{v}(x)} \sum_{r=0}^{n} D_{n, r} r !(1+v)_{r} \sum_{k=0}^{2 r} \frac{1}{k !} \frac{d^{k}}{d x^{k}}\left(x^{k} \rho_{v}(x)\right){ }_{3} F_{2}(-k, 1+v+r, 1+r ; 1+v, 1 ; 1),
$$

where $a_{n, 0}$ is defined by (4.33) and $D_{n}, D_{n, r}$ by (4.29), (4.30), respectively.

Corollary 2. Orthogonal polynomials $P_{n}$ have the following representation

$$
P_{n}(x)=-\frac{a_{n, 0}}{D_{n}} \sum_{r=0}^{n} D_{n, r} r !(1+v)_{r}\left[\sum_{k=0}^{r} \frac{A_{k, k-1}(x)}{(2 k) !}{ }_{3} F_{2}(-2 k, 1+v+r, 1+r ; 1+v, 1 ; 1)\right.
$$




$$
\left.+\sum_{k=0}^{r-1} \frac{A_{k, k}(x)}{(2 k+1) !} 3 F_{2}(-2 k-1,1+v+r, 1+r ; 1+v, 1 ; 1)\right],
$$

where $A_{k, k-1}, A_{k, k}$ are the type 1 multiple orthogonal polynomials of degree $k$, associated with the vector of weight functions $\left(\rho_{v}, \rho_{v+1}\right)$.

Proof. In fact, we write (4.42) in the form

$$
\begin{aligned}
P_{n}(x)= & -\frac{a_{n, 0}}{D_{n} \rho_{v}(x)} \sum_{r=0}^{n} D_{n, r} r !(1+v)_{r}\left[\sum_{k=0}^{r} \frac{1}{(2 k) !} \frac{d^{2 k}}{d x^{2 k}}\left(x^{2 k} \rho_{v}(x)\right)_{3} F_{2}(-2 k, 1+v+r, 1+r ; 1+v, 1 ; 1)\right. \\
& \left.+\sum_{k=0}^{r-1} \frac{1}{(2 k+1) !} \frac{d^{2 k+1}}{d x^{2 k+1}}\left(x^{2 k+1} \rho_{v}(x)\right)_{3} F_{2}(-2 k-1,1+v+r, 1+r ; 1+v, 1 ; 1)\right] .
\end{aligned}
$$

Meanwhile, appealing to the Rodrigues formulas for the type 1 multiple orthogonal polynomials associated with the vector of weight functions $\left(\rho_{v}, \rho_{v+1}\right)$ (see in [7]), it gives

$$
\begin{aligned}
& \frac{d^{2 k}}{d x^{2 k}}\left(x^{2 k} \rho_{v}(x)\right)=A_{k, k-1}(x) \rho_{v}(x)+B_{k, k-1}(x) \rho_{v+1}(x), \\
& \frac{d^{2 k+1}}{d x^{2 k+1}}\left(x^{2 k+1} \rho_{v}(x)\right)=A_{k, k}(x) \rho_{v}(x)+B_{k, k}(x) \rho_{v+1}(x),
\end{aligned}
$$

where $A_{k, k-1}, B_{k, k-1}$ are polynomials of degree $k, k-1$, respectively, and $A_{k, k}, B_{k, k}$ are polynomials of degree $k$. Therefore, substituting these expressions into (4.44), we obtain

$$
\begin{gathered}
P_{n}(x)=-\frac{a_{n, 0}}{D_{n}} \sum_{r=0}^{n} D_{n, r} r !(1+v)_{r}\left[\sum_{k=0}^{r} \frac{A_{k, k-1}(x)}{(2 k) !}{ }_{3} F_{2}(-2 k, 1+v+r, 1+r ; 1+v, 1 ; 1)\right. \\
\left.+\sum_{k=0}^{r-1} \frac{A_{k, k}(x)}{(2 k+1) !} 3 F_{2}(-2 k-1,1+v+r, 1+r ; 1+v, 1 ; 1)\right] \\
-\frac{a_{n, 0} \rho_{v+1}(x)}{D_{n} \rho_{v}(x)} \sum_{r=0}^{n} D_{n, r} r !(1+v)_{r}\left[\sum_{k=0}^{r} \frac{B_{k, k-1}(x)}{(2 k) !}{ }_{3} F_{2}(-2 k, 1+v+r, 1+r ; 1+v, 1 ; 1)\right. \\
\left.+\sum_{k=0}^{r-1} \frac{B_{k, k}(x)}{(2 k+1) !} 3 F_{2}(-2 k-1,1+v+r, 1+r ; 1+v, 1 ; 1)\right] .
\end{gathered}
$$

But the existence of a multiple orthogonal polynomial sequence with respect to the vector of weight functions $\left(\rho_{v}, \rho_{v+1}\right)$ implies the identity

$$
\begin{gathered}
\sum_{r=0}^{n} D_{n, r} r !(1+v)_{r}\left[\sum_{k=0}^{r} \frac{B_{k, k-1}(x)}{(2 k) !}{ }_{3} F_{2}(-2 k, 1+v+r, 1+r ; 1+v, 1 ; 1)\right. \\
\left.+\sum_{k=0}^{r-1} \frac{B_{k, k}(x)}{(2 k+1) !}{ }_{3} F_{2}(-2 k-1,1+v+r, 1+r ; 1+v, 1 ; 1)\right] \equiv 0
\end{gathered}
$$

which drives to (4.43) and completes the proof. 
Remark 2. In a similar manner orthogonal polynomials with the weight function $\rho_{v+1}(x) \rho_{v}(x)$ can be investigated. We leave this topic to the interested reader.

Finally, in this section we establish the generating function for polynomials $P_{n}$, which is defined as usually by the equality

$$
G(x, z)=\sum_{n=0}^{\infty} P_{n}(x) \frac{z^{n}}{n !}, \quad x>0, z \in \mathbb{C},
$$

where $|z|<h_{x}$ and $h_{x}>0$ is a convergence radius of the power series. To do this, we employ (3.1), (4.36) and (4.39), having the following equality from (4.45)

$$
G(x, z)=\frac{1}{\rho_{v}(x)} \sum_{n=0}^{\infty} \frac{z^{n}}{n !} \sum_{k=0}^{2 n} \frac{h_{2 n, k}}{k !} \frac{d^{k}}{d x^{k}}\left[x^{k} \rho_{v}(x)\right]=\frac{1}{\rho_{v}(x)} \sum_{n=0}^{\infty} \frac{z^{n}}{n !} \sum_{k=0}^{2 n} h_{2 n, k} \sum_{j=0}^{k}\left(\begin{array}{l}
k \\
j
\end{array}\right) \frac{(-1)^{j}}{(k-j) !} x^{j} \rho_{v-j}(x) .
$$

Meanwhile, the product $x^{j} \rho_{v-j}(x)$ is expressed in [3] as follows

$$
x^{j} \rho_{v-j}(x)=x^{j / 2} r_{j}(2 \sqrt{x} ; v) \rho_{v}(x)+x^{(j-1) / 2} r_{j-1}(2 \sqrt{x} ; v-1) \rho_{v+1}(x), \quad j \in \mathbb{N}_{0},
$$

where $r_{-1}(z ; v)=0$,

$$
x^{j / 2} r_{j}(2 \sqrt{x} ; v)=(-1)^{j} \sum_{i=0}^{[j / 2]}(v+i-j+1)_{j-2 i}(j-2 i+1)_{i} \frac{x^{i}}{i !} .
$$

Therefore this leads to the final expression of the generating function for the sequence $\left(P_{n}\right)_{n \in \mathbb{N}_{0}}$, namely,

$$
\begin{gathered}
G(x, z)=\sum_{n=0}^{\infty} \sum_{k=0}^{2 n} \sum_{j=0}^{k}\left(\begin{array}{c}
k \\
j
\end{array}\right) \frac{(-1)^{j} h_{2 n, k}}{n !(k-j) !} x^{j / 2} r_{j}(2 \sqrt{x} ; v) z^{n} \\
+\frac{\rho_{v+1}(x)}{\rho_{v}(x)} \sum_{n=0}^{\infty} \sum_{k=0}^{2 n} \sum_{j=0}^{k}\left(\begin{array}{c}
k \\
j
\end{array}\right) \frac{(-1)^{j} h_{2 n, k}}{n !(k-j) !} x^{(j-1) / 2} r_{j-1}(2 \sqrt{x} ; v-1) z^{n},
\end{gathered}
$$

where coefficients $h_{2 n, k}$ are defined by (4.40).

\section{NOTE ON THE MULTIPLE ORTHOGONAL POLYNOMIALS}

In this section we will exhibit two types of multiple orthogonal polynomials for the vector of weight functions $\left(\rho_{v}^{2}, \rho_{v+1}^{2}, \rho_{v} \rho_{v+1}\right), v>-1 / 2$ over $\mathbb{R}_{+}$with an additional factor $x^{\alpha}, \alpha>-1$. Precisely, we consider the type 1 polynomials $\left(A_{n}^{\alpha}, B_{n-1}^{\alpha}, C_{n-1}^{\alpha}\right), n \in \mathbb{N}$ of degree $n, n-1$, respectively, satisfying the orthogonality conditions

$$
\int_{0}^{\infty}\left[A_{n}^{\alpha}(x) \rho_{v}^{2}(x)+B_{n-1}^{\alpha}(x) \rho_{v+1}^{2}(x)+C_{n-1}^{\alpha}(x) \rho_{v}(x) \rho_{v+1}(x)\right] x^{\alpha+m} d x=0, m=0,1, \ldots, 3 n-1 .
$$

So, we have $3 n$ linear homogeneous equations with $3 n+1$ unknown coefficients of polynomials $A_{n}^{\alpha}, B_{n-1}^{\alpha}, C_{n-1}^{\alpha}$. Therefore we can find type 1 polynomials up to a multiplicative factor. Let us denote the function $q_{n, n-1, n-1}^{\alpha}$ for the convenience

$$
q_{n, n-1, n-1}^{\alpha}(x)=A_{n}^{\alpha}(x) \rho_{v}^{2}(x)+B_{n-1}^{\alpha}(x) \rho_{v+1}^{2}(x)+C_{n-1}^{\alpha}(x) \rho_{v}(x) \rho_{v+1}(x) .
$$

Type 2 polynomials $p_{n, n-1, n}^{\alpha}$ are monic polynomials of degree $3 n-1$ which satisfy the multiple orthogonality conditions 


$$
\begin{gathered}
\int_{0}^{\infty} p_{n, n-1, n}^{\alpha}(x) \rho_{v}^{2}(x) x^{\alpha+m} d x=0, \quad m=0,1, \ldots, n-1, \\
\int_{0}^{\infty} p_{n, n-1, n}^{\alpha}(x) \rho_{v+1}^{2}(x) x^{\alpha+m} d x=0, \quad m=0,1, \ldots, n-2, \\
\int_{0}^{\infty} p_{n, n-1, n}^{\alpha}(x) \rho_{v}(x) \rho_{v+1}(x) x^{\alpha+m} d x=0, \quad m=0,1, \ldots, n-1 .
\end{gathered}
$$

This gives $3 n-1$ linear equations with $3 n-1$ unknown coefficients since the leading coefficient is equal to 1. Hence it can be uniquely determined.

When $|v|<1 / 2$ the uniqueness of the representation (5.2) is validated by the following

Theorem 4. Let $n, m, l \in \mathbb{N}_{0},|v|<1 / 2, f_{n}, g_{m}, h_{l}$ be polynomials of degree at most $n, m$, l, respectively. Let

$$
f_{n}(x) \rho_{v}^{2}(x)+g_{m}(x) \rho_{v+1}^{2}(x)+h_{l}(x) \rho_{v}(x) \rho_{v+1}(x)=0
$$

for all $x>0$. Then $f_{n} \equiv 0, g_{m} \equiv 0, h_{l} \equiv 0$.

Proof. As is known (cf. [10]) the quotient $\rho_{v} / \rho_{v+1}$ is represented by the Ismail integral

$$
\frac{\rho_{v}(x)}{\rho_{v+1}(x)}=\frac{1}{\pi^{2}} \int_{0}^{\infty} \frac{s^{-1} d s}{(x+s)\left(J_{v+1}^{2}(2 \sqrt{s})+Y_{v+1}^{2}(2 \sqrt{s})\right)},
$$

where $J_{v}, Y_{v}$ are Bessel functions of the first and second kind, respectively [5], Vol. II. In fact, let $r \geq$ $\max \{n, m+1, l+1\}$. Hence, dividing (5.6) by $\rho_{v}(x) \rho_{v+1}(x)$ and using (3.6), we find

$$
f_{n}(x) \frac{\rho_{v}(x)}{\rho_{v+1}(x)}+x g_{m}(x) \frac{\rho_{v-1}(x)}{\rho_{v}(x)}+v g_{m}(x)+h_{l}(x)=0 .
$$

Then, differentiating $r$ times, it gives

$$
\frac{d^{r}}{d x^{r}}\left[f_{n}(x) \frac{\rho_{v}(x)}{\rho_{v+1}(x)}\right]+\frac{d^{r}}{d x^{r}}\left[x g_{m}(x) \frac{\rho_{v-1}(x)}{\rho_{v}(x)}\right]=0 .
$$

Assuming $f_{n}(x)=\sum_{k=0}^{n} f_{n, k} x^{k}$ and employing (5.7), the first term on the left-hand side of (5.9) can be treated as follows

$$
\begin{aligned}
\frac{d^{r}}{d x^{r}} & {\left[f_{n}(x) \frac{\rho_{v}(x)}{\rho_{v+1}(x)}\right]=\frac{1}{\pi^{2}} \frac{d^{r}}{d x^{r}} \sum_{k=0}^{n} f_{n, k} x^{k} \int_{0}^{\infty} e^{-x y} d y \int_{0}^{\infty} \frac{e^{-s y} s^{-1} d s}{J_{v+1}^{2}(2 \sqrt{s})+Y_{v+1}^{2}(2 \sqrt{s})} } \\
& =\frac{1}{\pi^{2}} \sum_{k=0}^{n} f_{n, k}(-1)^{k} \frac{d^{r}}{d x^{r}} \int_{0}^{\infty} \frac{d^{k}}{d y^{k}}\left[e^{-x y}\right] d y \int_{0}^{\infty} \frac{e^{-s y} s^{-1} d s}{J_{v+1}^{2}(2 \sqrt{s})+Y_{v+1}^{2}(2 \sqrt{s})} \\
& =\frac{1}{\pi^{2}} \sum_{k=0}^{n} f_{n, k}(-1)^{k} \int_{0}^{\infty} \frac{\partial^{k+r}}{\partial y^{k} \partial x^{r}}\left[e^{-x y}\right] d y \int_{0}^{\infty} \frac{e^{-s y} s^{-1} d s}{J_{v+1}^{2}(2 \sqrt{s})+Y_{v+1}^{2}(2 \sqrt{s})} \\
& =\frac{1}{\pi^{2}} \sum_{k=0}^{n} f_{n, k}(-1)^{k+r} \int_{0}^{\infty} \frac{d^{k}}{d y^{k}}\left[y^{r} e^{-x y}\right] d y \int_{0}^{\infty} \frac{e^{-s y} s^{-1} d s}{J_{v+1}^{2}(2 \sqrt{s})+Y_{v+1}^{2}(2 \sqrt{s})},
\end{aligned}
$$

where the differentiation under the integral sign is possible via the absolute and uniform convergence. Now, we integrate $k$ times by parts in the outer integral with respect to $y$ on the right-hand side of the latter equality, eliminating the integrated terms due to the choice of $r$, and then differentiate under the integral sign in the inner integral with respect to $s$ owing to the same arguments, to obtain 


$$
\frac{d^{r}}{d x^{r}}\left[f_{n}(x) \frac{\rho_{v}(x)}{\rho_{v+1}(x)}\right]=\frac{1}{\pi^{2}} \int_{0}^{\infty} y^{r} e^{-x y} \int_{0}^{\infty} \frac{e^{-s y} s^{-1}}{J_{v+1}^{2}(2 \sqrt{s})+Y_{v+1}^{2}(2 \sqrt{s})}\left(\sum_{k=0}^{n} f_{n, k}(-1)^{k+r} s^{k}\right) d s .
$$

In the same fashion the second term in (5.9) is worked out to find $\left(g_{m}(x)=\sum_{k=0}^{m} g_{m, k} x^{k}\right)$

$$
\frac{d^{r}}{d x^{r}}\left[x g_{m}(x) \frac{\rho_{v-1}(x)}{\rho_{v}(x)}\right]=\frac{1}{\pi^{2}} \int_{0}^{\infty} y^{r} e^{-x y} \int_{0}^{\infty} \frac{e^{-s y}}{J_{v}^{2}(2 \sqrt{s})+Y_{v}^{2}(2 \sqrt{s})}\left(\sum_{k=0}^{m} g_{m, k}(-1)^{k+r+1} s^{k}\right) d s .
$$

Substituting (5.10), (5.11) into (5.9) and cancelling twice the Laplace transform via its injectivity for integrable and continuous functions [6], we arrive at the equality

$$
x g_{m}(-x)\left[J_{v+1}^{2}(2 \sqrt{x})+Y_{v+1}^{2}(2 \sqrt{x})\right]+f_{n}(-x)\left[J_{v}^{2}(2 \sqrt{x})+Y_{v}^{2}(2 \sqrt{x})\right]=0, x>0 .
$$

The sum of squares of Bessel functions in brackets is called the Nicholson kernel, which has the MellinBarnes representation by virtue of Entry 8.4.20.35 in [5], Vol. III

$$
\begin{gathered}
x^{k}\left[J_{v}^{2}(2 \sqrt{x})+Y_{v}^{2}(2 \sqrt{x})\right]=\frac{2^{1-2 k} \cos (\pi v)}{2 \pi^{7 / 2} i} \int_{\gamma-i \infty}^{\gamma+i \infty} \Gamma(s+k) \Gamma(s+k+v) \Gamma(s+k-v) \\
\times \Gamma\left(\frac{1}{2}-s-k\right)(4 x)^{-s} d s, \quad|v|-k<\gamma<\frac{1}{2}-k .
\end{gathered}
$$

Then, using on the right-hand side of (5.13) the reflection formula for gamma function [1], Vol. I, it can be written as follows

$$
\begin{aligned}
& \frac{2^{1-2 k} \cos (\pi v)}{2 \pi^{7 / 2} i} \int_{\gamma-i \infty}^{\gamma+i \infty} \Gamma(s+k) \Gamma(s+k+v) \Gamma(s+k-v) \Gamma\left(\frac{1}{2}-s-k\right)(4 x)^{-s} d s \\
= & \frac{2^{1-2 k}(-1)^{k} \cos (\pi v)}{2 \pi^{7 / 2} i} \int_{\gamma-i \infty}^{\gamma+i \infty} \Gamma(s+k) \Gamma(s+k+v) \Gamma(s+k-v) \frac{\Gamma(1 / 2-s)}{(1 / 2+s)_{k}}(4 x)^{-s} d s .
\end{aligned}
$$

Our goal now is to shift the contour to the right to make integration along the straight line with $|v|<\operatorname{Res}<$ $1 / 2$. To do this we should take into account the residues at $k$ simple poles $s_{m}=-1 / 2-m, m=0,1, \ldots, k-1$ which have the values

$$
\begin{gathered}
\operatorname{Res}_{s=s_{m}}\left(\frac{\Gamma(s+k) \Gamma(s+k+v) \Gamma(s+k-v) \Gamma(1 / 2-s)}{(1 / 2+s)(3 / 2+s) \ldots(s+k-1 / 2)}(4 x)^{-s}\right) \\
=\frac{\Gamma\left(s_{m}+k\right) \Gamma\left(s_{m}+k+v\right) \Gamma\left(s_{m}+k-v\right) \Gamma\left(1 / 2-s_{m}\right)}{\left(1 / 2+s_{m}\right)\left(1 / 2+s_{m}+1\right) \ldots\left(1 / 2+s_{m}+m-1\right)\left(1 / 2+s_{m}+m+1\right) \ldots\left(1 / 2+s_{m}+k-1\right)}(4 x)^{-s_{m}} \\
=\frac{(-1)^{m}(4 x)^{1 / 2+m}}{(k-m-1) !} \Gamma(k-m-1 / 2) \Gamma(k-m-1 / 2+v) \Gamma(k-m-1 / 2-v) .
\end{gathered}
$$

Therefore we get from (5.14)

$$
\begin{aligned}
& \frac{2^{1-2 k} \cos (\pi v)}{2 \pi^{7 / 2} i} \int_{\gamma-i \infty}^{\gamma+i \infty} \Gamma(s+k) \Gamma(s+k+v) \Gamma(s+k-v) \Gamma\left(\frac{1}{2}-s-k\right)(4 x)^{-s} d s \\
= & \frac{2^{1-2 k}(-1)^{k} \cos (\pi v)}{2 \pi^{7 / 2} i} \int_{\mu-i \infty}^{\mu+i \infty} \Gamma(s+k) \Gamma(s+k+v) \Gamma(s+k-v) \frac{\Gamma(1 / 2-s)}{(1 / 2+s)_{k}}(4 x)^{-s} d s
\end{aligned}
$$




$$
-\frac{4^{1-k} \sqrt{x} \cos (\pi v)}{\pi^{5 / 2}} \sum_{m=0}^{k-1} \frac{(-1)^{k+m}(4 x)^{m}}{(k-m-1) !} \Gamma(k-m-1 / 2) \Gamma(k-m-1 / 2+v) \Gamma(k-m-1 / 2-v),
$$

where $|v|<\mu<1 / 2$. Now, recalling Parseval's equality for the Mellin transform and Entries 8.4.2.5, 8.4.23.27 in [5], Vol. III, we derive from (5.13), (5.15)

$$
\begin{gathered}
x^{k}\left[J_{v}^{2}(2 \sqrt{x})+Y_{v}^{2}(2 \sqrt{x})\right]=\frac{2^{3-2 k}(-1)^{k} \sqrt{x}}{\pi^{3}} \cos (\pi v) \int_{0}^{\infty} K_{v}^{2}(\sqrt{t}) \frac{t^{k-1 / 2}}{4 x+t} d t \\
-\frac{4^{1-k} \sqrt{x} \cos (\pi v)}{\pi^{5 / 2}} \sum_{m=0}^{k-1} \frac{(-1)^{k+m}(4 x)^{m}}{(k-m-1) !} \Gamma(k-m-1 / 2) \Gamma(k-m-1 / 2+v) \Gamma(k-m-1 / 2-v) .
\end{gathered}
$$

Analogously, we find

$$
\begin{gathered}
x^{k+1}\left[J_{v+1}^{2}(2 \sqrt{x})+Y_{v+1}^{2}(2 \sqrt{x})\right]=\frac{2^{1-2 k}(-1)^{k} \sqrt{x}}{\pi^{3}} \cos (\pi v) \int_{0}^{\infty} K_{v+1}^{2}(\sqrt{t}) \frac{t^{k+1 / 2}}{4 x+t} d t \\
-\frac{4^{-k} \sqrt{x} \cos (\pi v)}{\pi^{5 / 2}} \sum_{m=0}^{k} \frac{(-1)^{k+m+1}(4 x)^{m}}{(k-m) !} \Gamma(k-m+1 / 2) \Gamma(k-m+1 / 2+v) \Gamma(k-m+1 / 2-v) .
\end{gathered}
$$

Substituting expressions (5.16), (5.17) in (5.12), we derive after straightforward simplifications

$$
\begin{gathered}
\int_{0}^{\infty} K_{v+1}^{2}(2 \sqrt{t}) g_{m}(t) \frac{\sqrt{t}}{x+t} d t+\int_{0}^{\infty} K_{v}^{2}(2 \sqrt{t}) f_{n}(t) \frac{d t}{\sqrt{t}(x+t)} \\
+\sqrt{\pi} \sum_{k=0}^{m} \sum_{j=0}^{k} \frac{(-x)^{j}}{4^{k-j}(k-j) !} \Gamma(k-j+1 / 2) \Gamma(k-j+1 / 2+v) \Gamma(k-j+1 / 2-v) \\
-\sqrt{\pi} \sum_{k=0}^{n-1} \sum_{j=0}^{k} \frac{(-x)^{j}}{4^{k-j}(k-j) !} \Gamma(k-j+1 / 2) \Gamma(k-j+1 / 2+v) \Gamma(k-j+1 / 2-v)=0, x>0 .
\end{gathered}
$$

Last two terms in (5.18) are polynomials of degree $m, n-1$, respectively. Hence, differentiating through $r_{1} \geq \max \{n, m+1\}$ times by $x$, we obtain

$$
\frac{d^{r_{1}}}{d x^{r_{1}}} \int_{0}^{\infty} K_{v+1}^{2}(2 \sqrt{t}) g_{m}(t) \frac{\sqrt{t}}{x+t} d t+\frac{d^{r_{1}}}{d x^{r_{1}}} \int_{0}^{\infty} K_{v}^{2}(2 \sqrt{t}) f_{n}(t) \frac{d t}{\sqrt{t}(x+t)}=0 .
$$

The left-hand side of (5.19) is the $r_{1}$-th derivative of the sum of two Stieltjes transforms which are, in turn, two fold Laplace transforms. Consequently, fulfilling the differentiation under the integral sign in (5.19) as above owing to the absolute and uniform convergence by $x \geq x_{0}>0$, we cancel Laplace transforms of integrable functions via the injectivity. Then with (2.2) it yields the equality

$$
f_{n}(x) \rho_{v}^{2}(x)+g_{m}(x) \rho_{v+1}^{2}(x)=0, \quad x>0 .
$$

Comparing with (5.6), we see that $h_{l} \equiv 0$. Further, identity (5.20) implies that $f_{n}, g_{m}$ have the same positive roots, if any. Let $x>0$ be not a root of $g_{m}$. Then dividing (5.20) by $g_{m}$ and making a differentiation, we get

$$
-2 \rho_{v}(x) \rho_{v-1}(x) \frac{f_{n}(x)}{g_{m}(x)}+\rho_{v}^{2}(x) \frac{f_{n}^{\prime}(x) g_{m}(x)-f_{n}(x) g_{m}^{\prime}(x)}{g_{m}^{2}(x)}-2 \rho_{v+1}(x) \rho_{v}(x)=0 .
$$


Since $\rho_{v}(x)>0$, we divide the previous equation by $\rho_{v}$, multiply by $x, g_{m}^{2}$ and employ (3.6) to find

$$
-2 g_{m}(x) \rho_{v+1}(x)\left(f_{n}(x)+x g_{m}(x)\right)+\rho_{v}(x)\left(2 v f_{n}(x) g_{m}(x)+x\left(f_{n}^{\prime}(x) g_{m}(x)-f_{n}(x) g_{m}^{\prime}(x)\right)\right)=0 .
$$

However, the existence of the type 1 multiple orthogonal polynomials with respect to the vector of weight functions $\left(\rho_{v}, \rho_{v+1}\right)$ suggests the equalities

$$
g_{m}(x)\left(f_{n}(x)+x g_{m}(x)\right) \equiv 0, \quad 2 v f_{n}(x) g_{m}(x)+x\left(f_{n}^{\prime}(x) g_{m}(x)-f_{n}(x) g_{m}^{\prime}(x)\right) \equiv 0 .
$$

So, if $g_{m} \equiv 0$, it proves the theorem. Otherwise $f_{n}(x)+x g_{m}(x) \equiv 0$, and with the second equation in (5.22) we have $x(2 v+1) g_{m}^{2}(x) \equiv 0$. Thus $g_{m} \equiv 0$ and $f_{n} \equiv 0$ from (5.20). Theorem 4 is proved.

Remark 3. The choice of $v$ is important. For instance, for $v=-1 / 2$ the theorem fails. This can be seen, taking $f_{n}(x) \equiv-x, g_{m}(x) \equiv 1, h_{l}(x) \equiv 0$.

Theorem 5. Let $v \in[0,1 / 2)$. For every $\alpha>0$

$$
\frac{d}{d x}\left[x^{\alpha} q_{n, n-1, n-1}^{\alpha}(x)\right]=x^{\alpha-1} q_{n, n-1, n}^{\alpha-1}(x)
$$

and the following differential recurrence relations hold

$$
\begin{gathered}
A_{n}^{\alpha-1}(x)=(\alpha+2 v) A_{n}^{\alpha}(x)+x\left[A_{n}^{\alpha}(x)\right]^{\prime}-x C_{n-1}^{\alpha}(x), \\
B_{n-1}^{\alpha-1}(x)=\alpha B_{n-1}^{\alpha}(x)+x\left[B_{n-1}^{\alpha}(x)\right]^{\prime}-C_{n-1}^{\alpha}(x), \\
C_{n}^{\alpha-1}(x)=(\alpha+v) C_{n-1}^{\alpha}(x)+x\left[C_{n-1}^{\alpha}(x)\right]^{\prime}-2 A_{n}^{\alpha}(x)-2 x B_{n-1}^{\alpha}(x) .
\end{gathered}
$$

Proof. From (5.1), (5.2) and integration by parts we get

$$
\int_{0}^{\infty} q_{n, n-1, n-1}^{\alpha}(x) x^{\alpha+m} d x=-\frac{1}{m+1} \int_{0}^{\infty} \frac{d}{d x}\left[x^{\alpha} q_{n, n-1, n-1}^{\alpha}(x)\right] x^{m+1} d x=0,
$$

where the integrated terms vanish for every $\alpha>-1$ via asymptotic behavior (2.4), (2.5). Hence (5.1) suggests the equality

But, evidently,

$$
\int_{0}^{\infty} \frac{d}{d x}\left[x^{\alpha} q_{n, n-1, n-1}^{\alpha}(x)\right] x^{m} d x=0, \quad m=1, \ldots, 3 n
$$

$$
\int_{0}^{\infty} \frac{d}{d x}\left[x^{\alpha} q_{n, n-1, n-1}^{\alpha}(x)\right] d x=0, \quad \alpha>0
$$

Therefore

$$
\int_{0}^{\infty} \frac{d}{d x}\left[x^{\alpha} q_{n, n-1, n-1}^{\alpha}(x)\right] x^{m} d x=0, \quad m=0, \ldots, 3 n .
$$

Now, working out the differentiation in (5.27), involving (3.5), (3.6), we find

$$
\frac{d}{d x}\left[x^{\alpha} q_{n, n-1, n-1}^{\alpha}(x)\right]=\alpha x^{\alpha-1} q_{n, n-1, n-1}^{\alpha}(x)+x^{\alpha-1}\left(x\left[A_{n}^{\alpha}(x)\right]^{\prime} \rho_{v}^{2}(x)+x\left[B_{n-1}^{\alpha}(x)\right]^{\prime} \rho_{v+1}^{2}(x)+x\left[C_{n-1}^{\alpha}(x)\right]^{\prime} \rho_{v}(x) \rho_{v+1}(x)\right.
$$




$$
\begin{gathered}
+2 A_{n}^{\alpha}(x) \rho_{v}(x)\left(v \rho_{v}(x)-\rho_{v+1}(x)\right)-2 x B_{n-1}^{\alpha}(x) \rho_{v+1}(x) \rho_{v}(x) \\
\left.-x C_{n-1}^{\alpha}(x) \rho_{v}^{2}(x)+C_{n-1}^{\alpha}(x) \rho_{v+1}(x)\left(v \rho_{v}(x)-\rho_{v+1}(x)\right)\right) .
\end{gathered}
$$

Thus

$$
\frac{d}{d x}\left[x^{\alpha} q_{n, n-1, n-1}^{\alpha}(x)\right]=x^{\alpha-1}\left[A_{n}^{\alpha-1}(x) \rho_{v}^{2}(x)+B_{n-1}^{\alpha-1}(x) \rho_{v+1}^{2}(x)+C_{n}^{\alpha-1}(x) \rho_{v}(x) \rho_{v+1}(x)\right],
$$

where $A_{n}^{\alpha-1}(x), B_{n-1}^{\alpha-1}(x), C_{n}^{\alpha-1}(x)$ are polynomials of degree at most $n, n-1, n$, respectively, being defined by formulas (5.24), (5.25), (5.26). The linear homogeneous system (5.27) of $3 n+1$ equations contains $3 n+2$ unknowns. Therefore up to a constant, choosing to be one, the left-hand side of (5.28) is equal to $x^{\alpha-1} q_{n, n-1, n}^{\alpha-1}(x)$, and the representation (5.28) is unique by virtue of Theorem 4. This proves (5.23) and completes the proof of Theorem 5 .

Remark 4. The same analysis for the function $q_{n, n-1, n}^{\alpha}(x)$ does not work. In fact, in this case we derive analogously

$$
\int_{0}^{\infty} \frac{d}{d x}\left[x^{\alpha} q_{n, n-1, n}^{\alpha}(x)\right] x^{m} d x=0, \quad m=0, \ldots, 3 n+1 .
$$

However, working out the differentiation, we will get polynomials of degree at most $n+1, n, n$, respectively, which implies $3 n+4$ unknowns for $3 n+2$ equations (the so-called quasi multiple orthogonal case.)

Finally, we establish the differentiation property for the type 2 multiple orthogonal polynomials $p_{n, n-1, n}^{\alpha}$ (or 3-orthogonal polynomials).

Theorem 6. For every $v \geq 0, \alpha>-1$

$$
\frac{d}{d x}\left[p_{n, n-1, n}^{\alpha}(x)\right]=(3 n-1) p_{n, n-1, n-1}^{\alpha+1}(x) .
$$

Proof. Recalling (3.5), (3.6) and asymptotic behavior of the weight functions (2.4), (2.5), we integrate by parts in (5.3), eliminating the integrated terms, to deduce

$$
\int_{0}^{\infty} \frac{d}{d x}\left[p_{n, n-1, n}^{\alpha}(x)\right] \rho_{v}^{2}(x) x^{\alpha+1+m} d x=0, \quad m=0,1, \ldots, n-1 .
$$

Concerning equalities (5.4), (5.5), it corresponds the following ones

$$
\begin{gathered}
\int_{0}^{\infty} \frac{d}{d x}\left[p_{n, n-1, n}^{\alpha}(x)\right] \rho_{v+1}^{2}(x) x^{\alpha+1+m} d x=0, \quad m=0,1, \ldots, n-2, \\
\int_{0}^{\infty} \frac{d}{d x}\left[p_{n, n-1, n}^{\alpha}(x)\right] \rho_{v}(x) \rho_{v+1}(x) x^{\alpha+1+m} d x=0, \quad m=0,1, \ldots, n-2 .
\end{gathered}
$$

Now $\left[p_{n, n-1, n}^{\alpha}(x)\right]^{\prime}$ is a polynomial of degree $3 n-2$ with leading coefficient $3 n-1$ and by equalities (5.30), (5.31), (5.32) it satisfies orthogonality conditions (5.3), (5.4), (5.5) for the type 2 multiple orthogonal polynomial $p_{n, n-1, n-1}^{\alpha+1}(x)$. Hence we get (5.29) by unicity. Theorem 6 is proved. 


\section{REFERENCES}

1. A. Erdélyi,W. Magnus, F. Oberhettinger, and F.G. Tricomi, Higher Transcendental Functions,Vols. I and II, McGraw-Hill, New York, London, Toronto, 1953.

2. Yu.A. Brychkov, O.I. Marichev, N.V. Savischenko, Handbook of Mellin Transforms. Advances in Applied Mathematics, CRC Press, Boca Raton, 2018.

3. E. Coussement, W. Van Assche, Some properties of multiple orthogonal polynomials associated with Macdonald functions, J. Comput. Appl. Math. 133 (2001), 253-261.

4. NIST Digital Library of Mathematical Functions. http://dlmf.nist.gov/, Release 1.0.17 of 2017-12-22. F. W. J. Olver, A. B. Olde Daalhuis, D. W. Lozier, B. I. Schneider, R. F. Boisvert, C. W. Clark, B. R. Miller and B. V. Saunders, eds.

5. A. P. Prudnikov, Yu. A. Brychkov and O.I. Marichev, Vol. I: Elementary Functions, Gordon and Breach, New York, London, 1986; Vol. II: Special Functions, Gordon and Breach, New York, London, 1986; Vol. III: More Special Functions, Gordon and Breach, New York, London, 1990.

6. E.C. Titchmarsh, An Introduction to the Theory of Fourier Integrals, Clarendon Press, Oxford, 1937.

7. W. Van Assche and S. Yakubovich, Multiple orthogonal polynomials associated with Macdonald functions, Integral Transforms and Special Functions, 9 (2000), N 3, 229- 244.

8. O.V. Viskov, H.M. Srivastava, New approaches to certain identities involving differential operators, J. Math. Anal. Appl. 186 (1994), 1-10.

9. S. Yakubovich and Yu. Luchko, The Hypergeometric Approach to Integral Transforms and Convolutions, Kluwer Academic Publishers, Mathematics and Applications. Vol.287, 1994.

10. S. Yakubovich, Orthogonal polynomials with ultra-exponential weight functions: an explicit solution to the Ditkin-Prudnikov problem, Constr. Approx. (2021). DOI 10.1007/s00365-020-09523-0.

\section{AdDendum}

Corrigendum to "A method of composition orthogonality and new sequences of orthogonal polynomials and functions for non-classical weights" [J. Math. Anal. Appl. 499(2021) 125032]. DOI of original article: https://doi.org/10.1016/j.jmaa.2021.125032

In the last section of the article one should consider the type 2 multiple monic orthogonal polynomials $p_{n+1, n, n+1}^{\alpha}$ of degree $3 n+2$ which satisfy the orthogonality conditions

$$
\begin{gathered}
\int_{0}^{\infty} p_{n+1, n, n+1}^{\alpha}(x) \rho_{v}^{2}(x) x^{\alpha+m} d x=0, \quad m=0,1, \ldots, n, \\
\int_{0}^{\infty} p_{n+1, n, n+1}^{\alpha}(x) \rho_{v+1}^{2}(x) x^{\alpha+m} d x=0, \quad m=0,1, \ldots, n-1, \\
\int_{0}^{\infty} p_{n+1, n, n+1}^{\alpha}(x) \rho_{v}(x) \rho_{v+1}(x) x^{\alpha+m} d x=0, \quad m=0,1, \ldots, n .
\end{gathered}
$$

This gives $3 n+2$ linear equations with $3 n+2$ unknown coefficients since the leading coefficient is equal to 1. Hence it can be uniquely determined. Theorem 6 reads as follows.

Theorem 6. For every $v \geq 0, \alpha>-1$

$$
\frac{d}{d x}\left[p_{n+1, n, n+1}^{\alpha}(x)\right]=(3 n+2) p_{n+1, n, n}^{\alpha+1}(x) .
$$

Proof. In fact, recalling (3.5), (3.6) and asymptotic behavior of the weight functions (2.4), (2.5), we integrate by parts in (1), eliminating the integrated terms, to deduce

$$
\int_{0}^{\infty} \frac{d}{d x}\left[p_{n+1, n, n+1}^{\alpha}(x)\right] \rho_{v}^{2}(x) x^{\alpha+1+m} d x=0, \quad m=0,1, \ldots, n .
$$


Concerning equalities (2), (3), it corresponds the following ones

$$
\begin{gathered}
\int_{0}^{\infty} \frac{d}{d x}\left[p_{n+1, n, n+1}^{\alpha}(x)\right] \rho_{v+1}^{2}(x) x^{\alpha+1+m} d x=0, \quad m=0,1, \ldots, n-1, \\
\int_{0}^{\infty} \frac{d}{d x}\left[p_{n+1, n, n+1}^{\alpha}(x)\right] \rho_{v}(x) \rho_{v+1}(x) x^{\alpha+1+m} d x=0, \quad m=0,1, \ldots, n-1 .
\end{gathered}
$$

Now $\left[p_{n+1, n, n+1}^{\alpha}\right]^{\prime}$ is a polynomial of degree $3 n+1$ with leading coefficient $3 n+2$ and by the latter equalities it satisfies orthogonality conditions (1), (2), (3) for the type 2 multiple orthogonal polynomial $p_{n+1, n, n}^{\alpha+1}$. Hence we get (4) by unicity and complete the proof.

The author would like to apologise for any inconvenience caused. 